\title{
Tuning drug release in polyester thin films: terminal end-groups determine specific rates of additive-free controlled drug release
}

\author{
Terry WJ Steele, Charlotte L Huang, Saranya Kumar, Aneesa Iskandar, Aw Baoxin, Freddy Yin Chiang Boey,
} Joachim SC Loo and Subbu S Venkatraman

\begin{abstract}
Modulating the drug release from polyester matrices independently of material properties would be beneficial to those designing biodegradable medical implants, such as drug delivery devices, stents and screws. However, the most common approaches use additives that often drastically alter the desired material properties. Recently, we have developed tools that allow gradient film formulations and high-throughput drug quantitation for the determination of parameter-specific correlations. We propose that modulated drug release can be obtained via additive-free mechanisms in polyesters by simply controlling polymer erosion through acidic terminal functional groups. Our results showed that drug release in poly(lactic-co-glycolic acid) (PLGA) formulations could be tuned to produce large ranges in drug release with relatively small changes in terminal acidic functional groups. For example, PLGA 53/47 thin films could be tuned to have $10-60 \%$ drug release at 14 days or $10-90 \%$ drug release at 20 days, depending on the PLGA/PLGA blend formulation and concentration of acidic terminal functional groups. A linear R-square correlation of up to 0.9 was observed for the acidic groups and percent drug release. Below a threshold of 1 part per thousand acidic groups, there was no increase in drug release, which has implications for polymer processing and film integrity. NPG Asia Materials (2013) 5, e46; doi:10.1038/am.2013.9; published online 19 April 2013
\end{abstract}

Keywords: drug delivery; fluorescein; gradient; knife casting; PLGA; screening

\section{INTRODUCTION}

Biodegradable polyesters, such as poly(lactic-co-glycolic acid) (PLGA), have been incorporated into a number of drug delivery medical devices because of their numerous advantages, that is, they are commercially available in a range of formulations, approved by the Food and Drug Administration for implants, and tend to be predictable in drug release and degradation. In this respect, polyesters are unique among the many raw materials available for drug delivery. Natural polymers, such as polysaccharides and proteins, have inherent advantages, but these materials lack simple methods for controlling mechanical properties and biodegradability. ${ }^{1}$ Polyesters have been successful in the release of therapeutics that span the range from hydrophilic macromolecules (for example, small interfering RNA, growth factors and vaccines) to hydrophobic small molecule drugs (see reviews Mundargi et al. ${ }^{2}$ and Rytting et al. ${ }^{3}$ ).

However, for each therapeutic delivered, the rate of drug delivery from PLGA needs to be modified to specific parameters based on the needs of the medical device. After choosing the geometry (thin film vs nano/microparticle), the formulation scientist must decide which strategy is best suited for tailoring the required drug delivery. The most common method is the addition of additives (for example, polyethylene glycol (PEG)). Although the addition of additives is the simplest option, additives have wide ranging effects on drug/matrix phase separations and can substantially change the material properties. $^{4-6}$

Ideally, the best matrices would be composed of pure polyester and use alternative methods to modulate drug release without the addition of additives. This narrows the parameters to molecular weight (MW), polydispersity or changes in the polyester matrix environment, such as polyester blends or varying the terminal functional groups. Blending has been commonly used in a number of applications. Two such examples include poly(D,L-lactic acid)/ PLGA (PLA/PLGA) for the delivery of betamethasone or $\operatorname{poly}(\varepsilon-$ caprolactone)/PLGA for cell adhesion studies. ${ }^{7,8}$ Similarly, protein and ganciclovir controlled release matrices using PLGA/PLGA blends that differed in their MW and terminal functional groups and small MW PLGA with a carboxylic acid functional group blended with a large MW PLGA with an ester terminal group have been reported. ${ }^{9,10}$ Others studies have used carboxylic acid functional groups within the PLGA to increase the rate of drug delivery. ${ }^{11,12}$ 
These studies suggest that PLGA matrices can be tailored via MW, terminal functional groups or both without the inclusion of additives. Despite these observations, no systematic study has been performed to quantify the influence on controlled drug release comparing both MW and terminal functional groups, especially for thin films used in medical devices. This lack of research is most likely due to the large number of film formulations that would need to be cast under specific conditions to determine the parameter-specific correlations. In addition, a high-throughput method is required to quantify the controlled release in a routine and timely manner.

Recently, our laboratory has developed such tools to allow tuning of PLGA controlled release using both MW and terminal functional groups, which can be characterized in a systematic, high-throughput method. We have previously published a method for gradient casting films with $\sim 1 \% \mathrm{~cm}^{-1}$ change in the PLGA/PEG and polypropylene composition. These gradient films allowed us to determine which additive was more efficient for tuning drug release and simultaneously evaluate how the various additives affected the mechanical properties. ${ }^{6}$ A high-throughput method was concurrently used to quantify the controlled release of model hydrophobic drugs with octanol/water partition coefficients $(\log \mathrm{P})$ of $\sim 4 .^{13}$

We proposed that the controlled release of PLGA could be tuned to specific controlled delivery rates through gradients by altering the MW and terminal functional groups, especially acid-terminal functional groups, of PLGA. For the terminal functional groups, we chose either a hydrophilic, more soluble carboxylic acid terminal group or the standard, hydrophobic ester terminal functional group. We have previously published the drug release of paclitaxel and fluorescein diacetate (FDAc) as well as the MW decay constant $\mathrm{k}$, mass loss, water absorption, differential scanning calorimetry scans, mechanical properties and the statistical correlations of five 'neat' PLGA films reported in the gradient formulations found in the article (that is, $\mathrm{P} 02 \mathrm{~A}, \mathrm{P} 02 \mathrm{E}$, P04A, P04E and P103E). ${ }^{14}$ This previously published manuscript also suggested that acid terminal end-groups could be used to tailor the drug release kinetics from PLGA blended thin films. These concepts are more thoroughly explored in the article.

\section{MATERIALS AND METHODS}

\section{Materials}

Poly (DL-lactide-co-glycolide) 53/47 (PLGA), which is summarized in Table 1 , was purchased from Purac (Gorinchem, The Netherlands). High-performance liquid chromatography grade dichloromethane and acetonitrile were purchased from Tritech Scientific Pte Ltd, Singapore. Deuterated chloroform

Table 1 Properties of poly (DL-lactide-co-glycolide) 53/47 (PLGA) used in gradient casting

\begin{tabular}{lcccc}
\hline & $\begin{array}{c}\text { Purac } \\
\text { PLGA 53/47 }\end{array}$ & $\begin{array}{c}\text { Intrinsic } \\
\text { viscosity } \\
\text { abbreviation }\end{array}$ & $\begin{array}{c}\text { Molecular } \\
\text { weight, } M W \\
\left(\mathrm{~d} / \mathrm{g}^{-1}\right)^{\mathrm{a}}\end{array}$ & $\begin{array}{c}\text { Terminal } \\
\text { functional }\end{array}$ \\
\hline P103E & PDLG 5010 & 1.03 & 110 & group \\
P04E & PDLG 5004 & 0.4 & 50 & $\begin{array}{c}\text { Methyl ester } \\
\text { Carboxylic }\end{array}$ \\
P04A & PDLG 5004A & 0.4 & 40 & $\begin{array}{c}\text { acid } \\
\text { P02E }\end{array}$ \\
P02A & PDLG 5002 & 0.2 & 20 & $\begin{array}{c}\text { Methyl ester } \\
\text { Carboxylic } \\
\text { acid }\end{array}$ \\
\hline
\end{tabular}

Abbreviations: PLGA, poly(lactic-co-glycolic acid); SEC, size exclusion chromatography. a Manufacturer supplied data.

${ }^{b}$ Calculated by multi-angle laser light scattering coupled to size exclusion chromatography (see section 'Quantitative analysis of PLGA mixtures by SEC-MALLS').
$\left(\mathrm{CDCl}_{3}+0.03 \% \mathrm{v} / \mathrm{v}\right.$ tetramethysilane $\mathrm{D} 99.8 \%+$ silver foil $)$ was purchased from Cambridge Isotope Laboratories, Andover, MA, USA. FDAc was purchased from TCI Japan, Singapore, Singapore. All of the other polar solvents used were of high-performance liquid chromatography grade and purchased from Sigma-Aldrich, Singapore, Singapore. All of the chemicals and materials were used as received.

\section{Gradient casting PLGA/PLGA blends into thin films}

The gradient films were produced according to previously published protocols with the modifications described here. ${ }^{6}$ Briefly, $20 \mathrm{ml}$ of the more viscous solution, $15 \% \mathrm{P} 103 \mathrm{E}$ (w/v dichloromethane), was poured into chamber $\mathrm{B}$, and the less viscous, lower MW PLGA was poured into chamber A (that is, $20 \mathrm{ml}$ $15 \%$ PLGA $5 \mathrm{kDa}$ acid terminated PLGA (w/v dichloromethane)). Each well contained $65 \mathrm{mg}$ (in $20 \mathrm{ml}, \sim 1 / 40 \mathrm{w} / \mathrm{w}$ of drug/polymer) of FDAc. The mixing in chamber B was performed using a battery-operated overhead stirrer (note: use only non-flammable solvents). Gradient solutions were peristaltically pumped (rate of approximately $20 \mathrm{ml} \mathrm{min}^{-1}$ ) directly into the $500-\mu \mathrm{m}$ thick film applicator in a fume hood. After vacuum drying, punch-outs that were $5 \mathrm{~mm}$ in diameter were taken at every 5 or $10 \mathrm{~cm}$ for analysis.

\section{PLGA, FDAc and thickness quantification by ${ }^{1} \mathrm{H}$ NMR}

The dried $(5 \mathrm{~mm}$ diameter $\times 3$ pieces $)$ punch-outs were dissolved in $1050 \pm 10 \mu \mathrm{g}(700 \mu \mathrm{l})$ of $\mathrm{CDCl}_{3}$, vortexed and centrifuged at $14000 \times \mathrm{g}$ for 5 min before transferring the supernatant into NMR tubes. The ${ }^{1} \mathrm{H}$ nuclear magnetic resonance (NMR) spectra were recorded on a Bruker Advance Spectrometer (Bruker, Billerica, MA, USA) at $400 \mathrm{MHz}$ using the tetramethysilane signal present in deuterated chloroform at $0.03 \%$ as an internal standard. ${ }^{1} \mathrm{H}$ NMR $\left(400 \mathrm{MHz}, \mathrm{CDCl}_{3}, \delta\right)$ 1.5-1.7 [bs, PLGA $3 \mathrm{H},-\mathrm{C}(=\mathrm{O})-\mathrm{CH}\left(\mathrm{CH}_{3}\right)-\mathrm{O}-$ $\left.\mathrm{C}(=\mathrm{O})-\mathrm{CH}_{2}-\mathrm{O}-\right], 4.6-5.0$ [bs, PLGA $2 \mathrm{H},-\mathrm{C}(=\mathrm{O})-\mathrm{CH}\left(\mathrm{CH}_{3}\right)-\mathrm{O}-\mathrm{C}(=\mathrm{O})-$ $\mathrm{CH}_{2}$-O-], 5.0-5.3 [bs, PLGA 1H, $-\mathrm{C}(=\mathrm{O})-\mathrm{CH}\left(\mathrm{CH}_{3}\right)-\mathrm{O}-\mathrm{C}(=\mathrm{O})-\mathrm{CH}_{2}-\mathrm{O}-$ ]. The ${ }^{1} \mathrm{H}$ NMR error was calculated using the combined s.d. (within each gradient composition) of the integrated $\mathrm{CHCl}_{3}$ peaks (weighing error) and the s.d. of the lactide/glycolide ratios (integration and machine error). The film thickness was estimated from the ${ }^{1} \mathrm{H}$ NMR composition data combined with the known densities of the PLGA and FDAc. The ${ }^{1} \mathrm{H}$ NMR generated volume (that is, $\left.1 \times 10^{7} \mu \mathrm{m}^{3}\right)$ was divided by the known surface area $(5 \mathrm{~mm}$ diameter punchout $\left.=1.96 \times 10^{5} \mu \mathrm{m}^{2}\right)$ to determine the thickness $(\sim 50 \mu \mathrm{m})$. For simplicity, the total PLGA cm${ }^{-2}$ can be divided by 122 to yield the thickness in microns.

\section{Quantitative analysis of PLGA mixtures by size exclusion} chromatography (SEC)-multiangle laser light scattering (MALLS) An Agilent 1100 series high-performance liquid chromatography pump complete with degasser and PLGel aqueous 50 (Polymer Standards Service, Mainz, Germany) in a $35^{\circ} \mathrm{C}$ temperature-controlled oven was connected inline with a Wyatt MiniDawn 3-angle light scattering detector and an Agilent 1100 refractive index detector (Agilent Technologies, Santa Clara, CA, USA). The elution buffer was tetrahydrafuran at a flow rate of $1.0 \mathrm{ml} \mathrm{min}^{-1}$. The injection volumes were typically $50 \mu$ l. The polymer mass was determined with a refractive index detector using a $\mathrm{dn} / \mathrm{dc}$ of 0.05 and the Wyatt ASTRA (version 5.19.1) software (Wyatt Technology, Santa Barbara, CA, USA). Redundant MW analysis was performed by injecting PEG standards of known MW to characterize the small molar mass oligomers $\left(<10000 \mathrm{~g} \mathrm{~mol}^{-1}\right)$ that exhibited minimal light scattering signals (Polymer Standards Service, Mainz, Germany).

\section{High-throughput screening of fluorescent dyes}

The high-throughput FDAc quantification has been previously published. ${ }^{13}$

\section{Data analysis}

Linear regressions and Pearson's correlations were calculated with Origin 8.5 SR1 (OriginLab, Northampton, MA, USA). Linear regression was determined using instrumental weighting. Pearson's correlations $(r)$ were determined with a minimum of $n=5$ data point comparisons and a two-tailed test of significance. Significance was determined as $P<0.05$. Analysis of covariance was used to determine the significance in the linear regression comparisons, with $P>0.95$ marked by an asterisk. 


\section{RESULTS}

Knife casting gradient PLGA/PLGA blends

The overall description, gradient caster dimensions and limitations of the technique have previously been described for gradient knife casting of PLGA/additive blended films. ${ }^{6}$ Only trivial changes in the methodology were used for the PLGA/PLGA blends (that is, addition of a peristaltic pump for more accurate flow control, $5 \mathrm{~mm}$ diameter punch-outs for drug release studies and shorter gradient lengths of $110-140 \mathrm{~cm}$ ). The shorter gradients were possible because of the thicker viscosity of the PLGA polymer solution compared with oligomer additives with similar $\mathrm{w} / \mathrm{w}$ ratios.

\section{${ }^{1} \mathrm{H}$ NMR quantitation of total PLGA and FDAc}

The total mass of PLGA and the amount of FDAc was determined with ${ }^{1} \mathrm{H}$ NMR. Previously, we were able to determine the ratios of PLGA and additive on dissolution in $\mathrm{CDCl}_{3}$ followed by NMR characterization. However, with the PLGA blends, no discernment was possible between varying PLGA MWs in the same sample via NMR analysis. The lactide and glycolide ${ }^{1} \mathrm{H}$ NMR peaks exhibit the same chemical shifts independent of the PLGA MW. For high MW PLGA, the peaks are broader, but the measurements of polydispersity and PLGA ratios by NMR were not possible. However, spiking one of the PLGA mixtures with trace amounts of a non-volatile additive with intense NMR signals, such as pyrene, would allow straightforward PLGA ratio determination. For this initial PLGA/PLGA gradient characterization, we chose to keep all additives to a minimum and no spiked PLGAs were used.

\section{SEC for P0XX/P103E ratio quantitation}

Calculation of the P0XX/P103E (P0XX refers to P02A, P02E, P04A or $\mathrm{P} 04 \mathrm{E}$ ) ratios was found to be considerably more laborious than originally envisioned. SEC was used for the determination of the $\mathrm{P} 0 \mathrm{XX} / \mathrm{P} 103 \mathrm{E}$ ratios. However, the hydrodynamic sizes of $\mathrm{P} 103 \mathrm{E}$ mixed with any P0XX were similar, yielding co-eluting 'camel back' peaks. Gaussian deconvolution analyses were either not repeatable, inaccurate or both. The co-elution was most prominent between the P103E and P04X (or 0.4 intrinsic viscosity) blends. Although the samples had different MWs, the polymer peaks eluted fairly close together, even with three SEC columns serially connected (data not shown). To overcome this obstacle, SEC deconvolution techniques were performed according to previously published protocols. ${ }^{15,16}$ Briefly, mathematical models were constructed from the SEC chromatograms of the five pure PLGA injections to predict the height ratios of the P0XX/P103E PLGA blends. After subtraction of the baseline, the pure PLGA elution peaks were normalized to $100 \mu \mathrm{g}$ mass. For example, to determine the 1:9 relative height ratio for the $\mathrm{P} 02 \mathrm{E} / \mathrm{P} 103 \mathrm{E}$ standard curve, $10 \%$ of the refractive index signal of the $100 \mu \mathrm{g}$ P02E baselined/normalized SEC chromatogram was added to $90 \%$ of the refractive index signal for the $100 \mu \mathrm{g}$ P103E baselined/normalized SEC chromatogram. In this method, a standard curve was constructed by plotting the relative peak height ratios as a function of the $\mathrm{P} 0 \mathrm{XX} / \mathrm{P} 103 \mathrm{E}$ ratios (see Figure 1). Then, the baseline was subtracted from the analyzed data, and the peak height ratio was converted to the P0XX/P103E ratios (normalizing the unknowns was not necessary). After testing three ratios of known composition, (P0XX/P103E ratios of 3:7, 5:5 and $7: 3)$, the maximum s.d. was found to be $\leqslant \pm 8 \%$ for the P04X and $\leqslant \pm 5 \%$ for the P02X blended films.
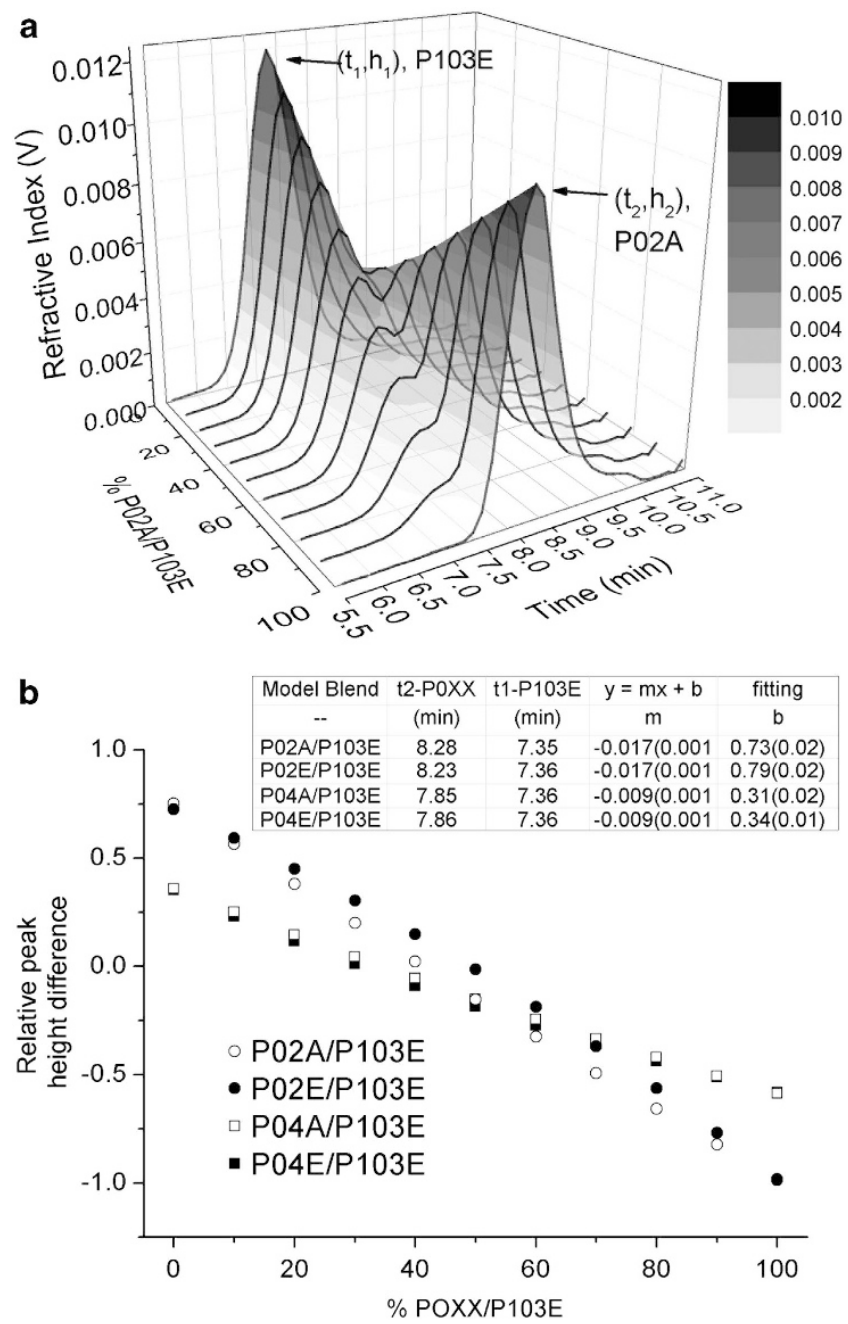

Figure 1 Mathematical deconvolution model for determining the ratios of the P0XX/P103E blends. (a) Construction of P02A/P103E simulated ratios (from 1:9 to 9:1) from pure P02A and P103E SEC chromatograms. (b) Linear regression of the relative peak height difference as a function of the POXX percentage. Relative peak height difference $=\left(h_{1} t_{1}-h_{2} t_{2}\right)$ / $\left(h_{1} t_{1}+h_{2} t_{2}\right) . h_{1}$ and $h_{2}$ are the baseline subtracted refractive index values at the respective $t_{1}$ and $t_{2}$ time points.

Optimal gradients of acid terminal end-groups depends on the MW of additive PLGA

With our gradient film casting method, a continuous increase in acid concentration can be synthesized into a single thin film. For the results reported herein, only four gradient thin films were cast, as shown in Scheme 1. This method allows for a more confident 'freezing' of the myriad parameters that can affect the synthesis of thin films or the final dried thin films themselves. The addition of PLGA with a terminal acid group must be balanced between two factors because an increased acid gradient can result in a wider range of polymer degradation with more variations possible for the subsequent drug release. However, the addition of small $(<5 \mathrm{kDa})$ PLGA oligomer acids would likely do little to catalyze drug release because they may quickly diffuse from the PLGA matrix. To achieve an acceptable balance of these factors, commercially available PLGA containing acid or ester functional groups with an intrinsic viscosity 


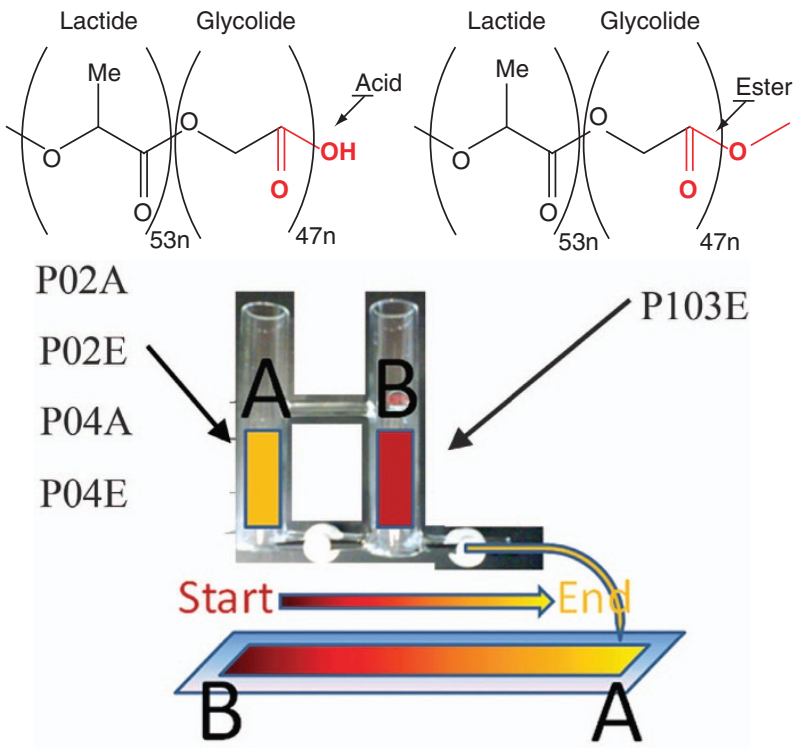

Scheme 1 Synthesis of POXX/P103E gradient thin films allowing tailored controlled release in the absence of additives.

of 0.2 and $0.4 \mathrm{dlg}^{-1}$ and MWs of $\sim 20$ and $50 \mathrm{kDa}$ were chosen, as shown in Table 1. P02A and P04A yield gradients that have a maximum of 8.9 and 3.3 p.p.t. (parts per thousand), respectively, of the terminal acid monomer to the sum of the lactide and glycolide monomers. As these acid gradients must be formed by the POXX polymers with $\mathrm{P} 103 \mathrm{E}$, the cumulative MW will also differ across the gradients. To separate the combined effects of MW and acid concentration on drug release, control gradient films of P02E or P04E were also cast with the P103E. These films were methyl ester terminated and contained no acid groups.

\section{Gradient comparisons and respective surface compositions of PLGAs and encapsulated drugs}

The average of the four gradient \% P0XX additives was $0.73 \pm 0.06 \%$ per $\mathrm{cm}$ when cast at a wet thickness of $500 \mu \mathrm{m}$. The small s.d. between the slopes suggests that the casting conditions were fairly reproducible, as shown in Figure 2. The gradient compositions in regards to molar mass (MW-weight-averaged, left axis, black) and \% POXX (right axis, gray) as a function of the gradient length are also compared in Figure 2. Note that the molar mass formulations under $50 \%$ were typically analyzed via PEG standards vs laser light scattering because of the small refractive index differential between PLGA and tetrahydrafuran. Figures $3 \mathrm{a}$ and $\mathrm{b}$ compare the differences in the PLGA gradients with 0.2 and $1.03 \mathrm{dlg}^{-1}$ intrinsic viscosity gradients that have acid (P02A) or ester (P02E) terminated functional groups. Interestingly, small differences were observed when the overall composition was compared for the acid and ester terminal groups. For the P02A or P04A total PLGA gradients in Figures 3a and c, the trends were slightly positive, with stable slopes compared with the P02E or P04E gradients in Figures $3 \mathrm{~b}$ and d. A common empirical observation in the synthesis of thin films was that a lower viscosity resulted in thinner films, as shown in Figures $3 \mathrm{~b}$ and $\mathrm{d}$. As viscosity was dependent on the MW of the polymer, the knife cast solutions became less viscous or thinner as the smaller MW polymer was mixed into the solutions, even though the polymer concentration remained at $10 \% \mathrm{w} / \mathrm{v}$. We speculate that the slightly positive and stable slopes observed in Figures $3 \mathrm{a}$ and $\mathrm{b}$ (total PLGA) may be due to intermolecular hydrogen bonding by the carboxylic acid end-group. The trend was slightly positive for the more carboxylic acid concentrated P02A then the P04A. The thickness (and therefore viscosity) did not decrease on MW dilution for these two polymers that were mixed with P103E. Such interactions have been observed in other polymer matrices containing acidified carboxylic acid h-donors and oxygen h-acceptors (for example, poly(methacrylic acid)/PEG complexes). ${ }^{17}$

\section{Controlled drug release of FDAc}

A remarkable increase in drug release was observed in the P02A gradients compared with the P02E gradients, as shown in Figures $4 \mathrm{a}$ and $\mathrm{b}$, respectively. Most of the P02E gradient formulations required longer than 20 days to release just $10 \%$ of the drug (Figure $4 \mathrm{~b}$ ). However, the P02A gradients displayed predictable shifts in drug release as the ratios of $\mathrm{P} 02 \mathrm{~A} / \mathrm{P} 103 \mathrm{E}$ increased over time. For example, depending on the P02A/P103E ratio, one could choose the formulation to have $25 \%$ drug release on any day between 10 and 21 days or a $50 \%$ drug release between 15 and 30 days. Such flexibility was not possible with the P02E gradients, suggesting that the MW dilution had little to do with the increase in drug release and expanded flexibility. Although not as pronounced, the P04A/P103E ratios displayed similar trends in increased drug release variability compared with P04E/P103E, as shown in Figures 4c and d.

One unforeseen result was found in the P04E gradients. In Figure 4, the $\%$ FDAc release had a negative correlation to the P04E percentage. The percentage of P04E in the film was not likely to be the dominant factor for the sudden increase in FDAc release. The most plausible explanation was the combined effects of a thicker film and the increase in the amount of P04E. The autocatalysis increased as the thickness of the films increased because of incomplete diffusion from the accumulation of acidic fragments. ${ }^{16}$ For example, for the starting conditions of $0 \%$ P04E with a relatively thick $70 \mu \mathrm{m}$ film (see Figure $4 \mathrm{~d}$, inset), only a $35 \%$ FDAc release was observed over 30 days, with the majority of the release occurring in the last few days of measurement. However, when the starting conditions were $20-50 \%$ P04E with the $\sim 50-\mu \mathrm{m}$ thick films, $75 \%$ drug release is observed. This release diminished to $35 \%$ once the thickness fell below $25 \mu \mathrm{m}$. Therefore, the autocatalytic effect was considerably diminished by diffusion of the acidified fragments (see Discussion section below).

\section{Correlation of percent drug release vs acid terminal end-group concentration}

Evaluating the amount of drug release over time is often a trial and error task with few tools that control and predict drug release kinetics. To measure the ability of the acid terminal end-groups to control the amount of drug release, the total drug release was plotted as a function of the percent additives of P02A, P04A and P02E, as shown in Figures $5 \mathrm{a}-\mathrm{c}$. The data suggest that strong Pearson's $\mathrm{r}$ correlations existed for the acid terminal end-group additives. In addition, this correlation grew stronger with time, until a plateau was reached (see Figure 5d). For the P04A gradients, the correlation decreased after 25 days because of drug depletion within the film matrix, as shown in Figure $5 \mathrm{~d}$ (open squares). When the ester terminal end-groups were similarly analyzed, they displayed a weak (P02E) or negative (P04E) correlation. As described in the previous section, the negative correlation likely arises from the increased thickness of the film.

The most useful for the formulation scientist was the observation that the drug release and additive/acid concentration exhibited a nearly linear dependence. For example, at days 10, 14 and 20 for the P02A polyester, linear fits of the total drug release as a function of the 
a
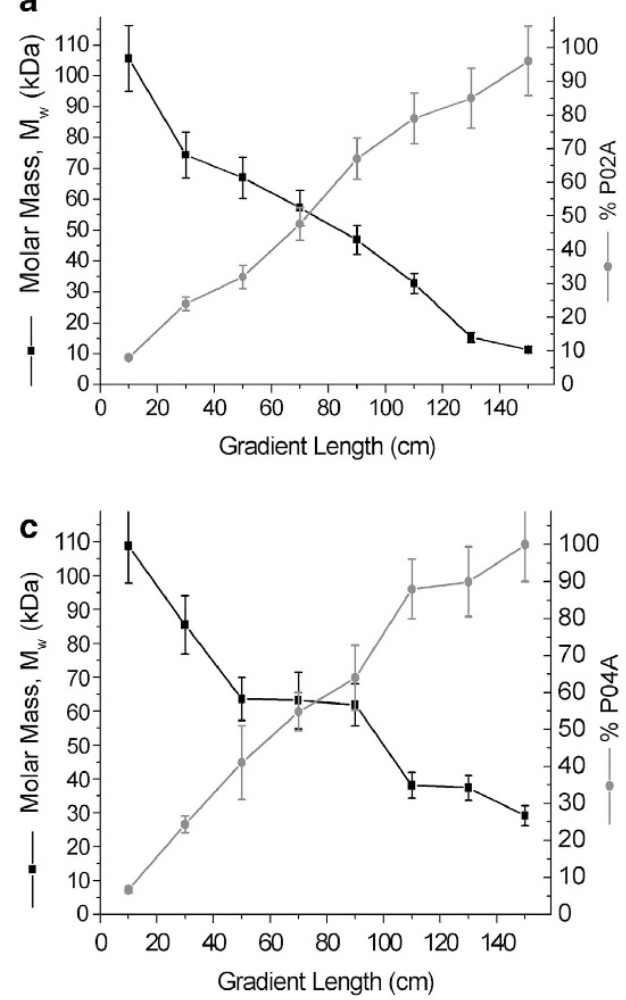
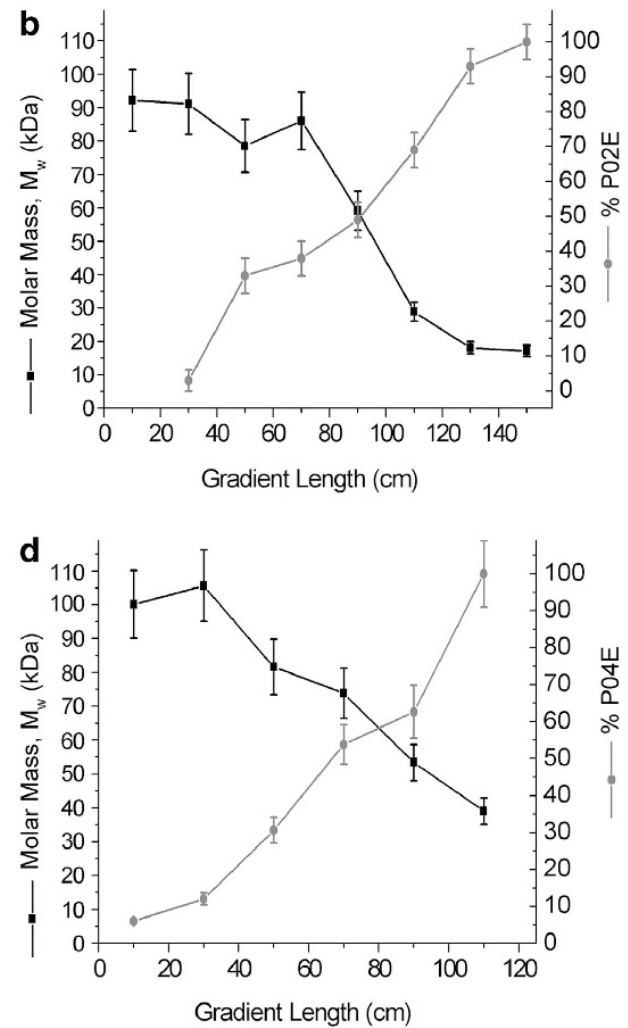

Figure 2 P02A and P103E gradient blend. Weight averaged molecular weight (MW) for the P02A/P103E blended film and the percentage of P02A at different gradient lengths. For simplicity, total PLGAcm ${ }^{-2}$ can be divided by 122 to yield the thickness in microns. (a) P02A/P103E gradient \% P02A slope $=(0.68 \pm 0.02) \quad \% \mathrm{~cm}^{-1} ; \quad$ (b) P02E/P103E gradient \% P02E slope $=(0.81 \pm 0.05) \quad \% \mathrm{~cm}^{-1} ; \quad$ (c) PO4A/P103E gradient \% P04A slope $=(0.75 \pm 0.03) \% \mathrm{~cm}^{-1}$; (d) P04E/P103E gradient \% PO4E slope $=(0.66 \pm 0.11) \% \mathrm{~cm}^{-1}$.
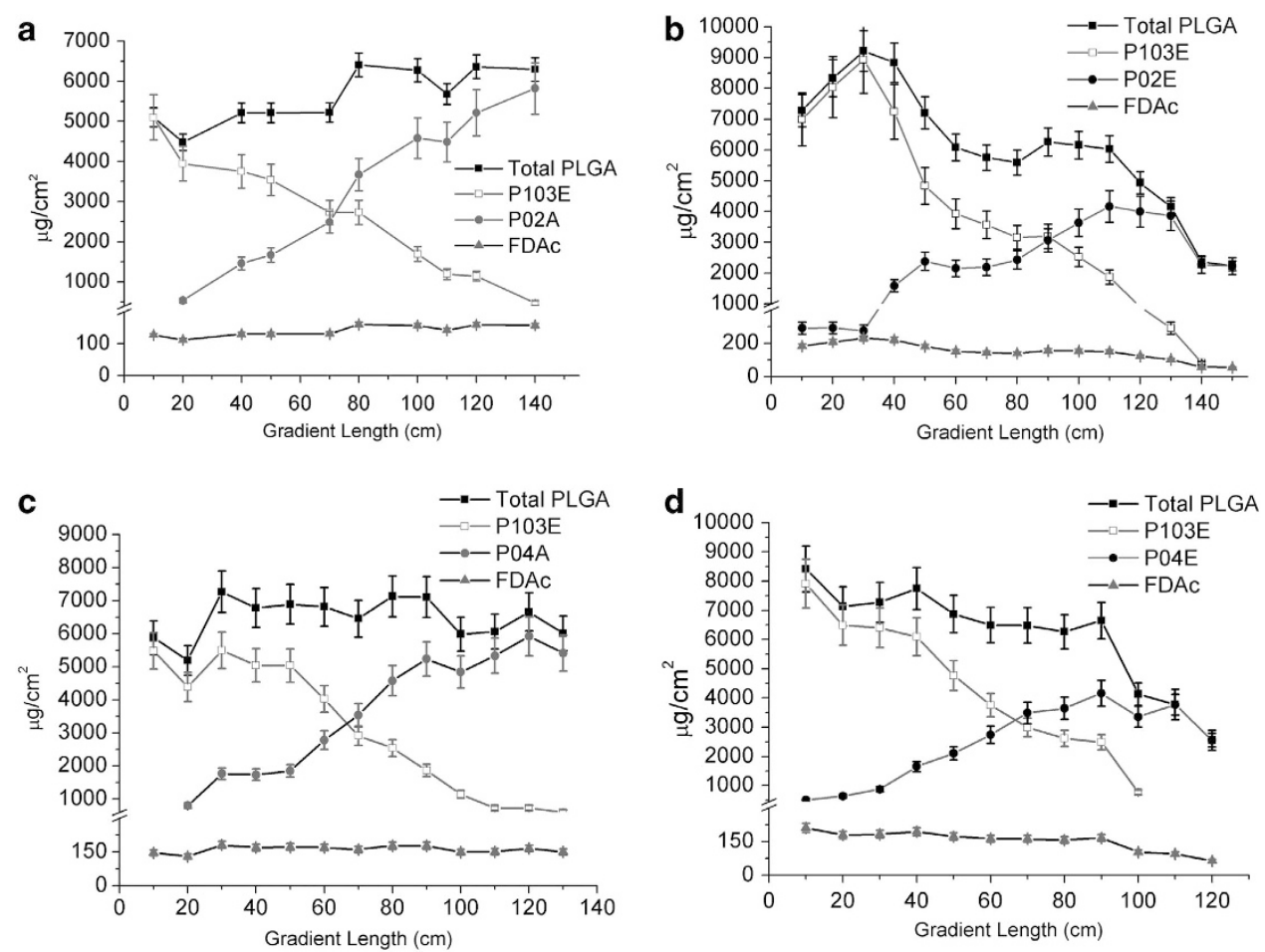

Figure 3 POXX/P103E blended film composition and their respective surface concentrations. Fluorescein diacetate (FDAc) was kept at a $\sim 1 / 40$ w/w FDAc/ poly(lactic-co-glycolic acid) (PLGA) ratio. Y axis break is from 400 to $1000 \mu \mathrm{gcm}^{-2}$. (a) P02A/P103E gradient; (b) P02E/P103E gradient; (c) P04A/P103E gradient; (d) P04E/P103E gradient. 

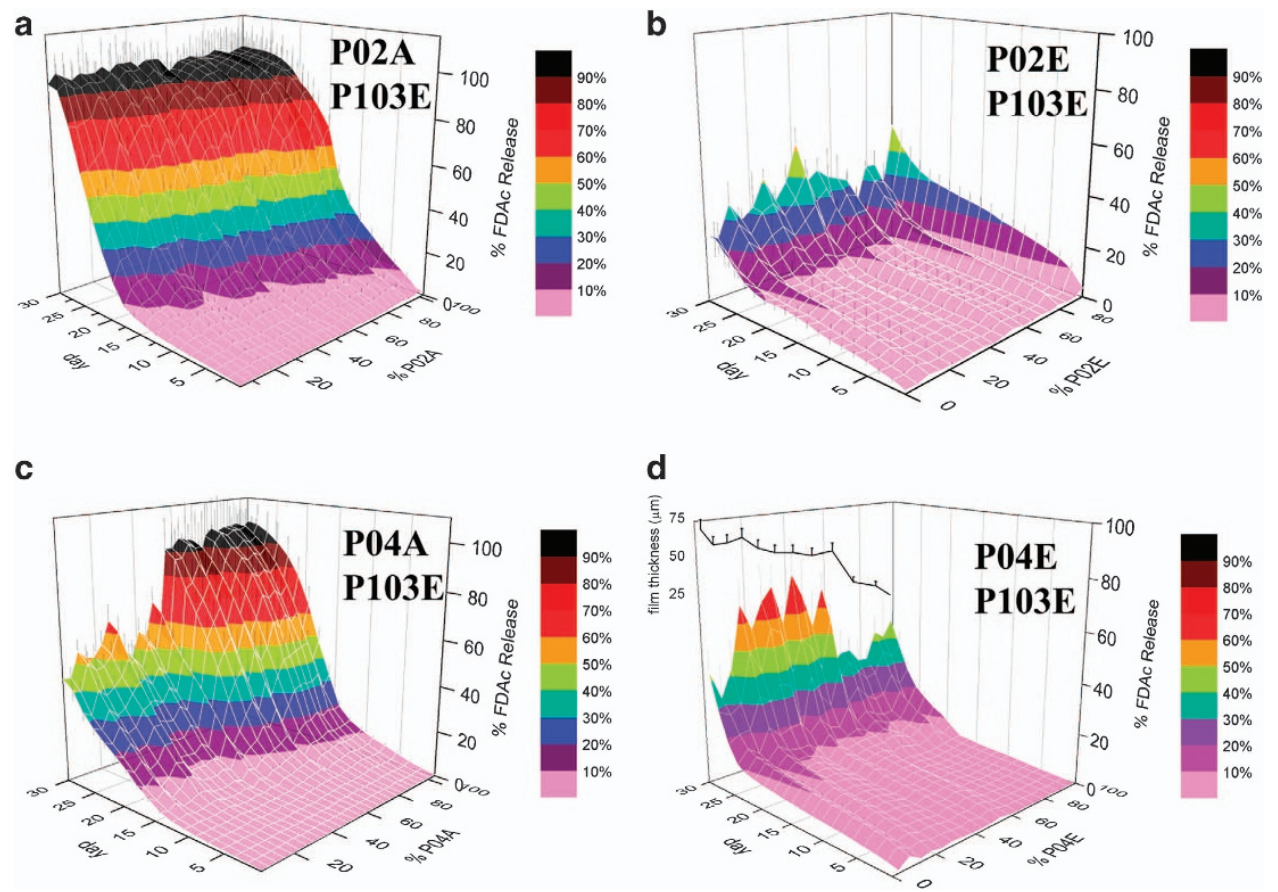

Figure 4 Cumulative release \% of fluorescein diacetate (FDAc; $z$ axis) as a function of time ( $X$ axis) and \% P0XX additive. (a) P02A/P103E gradient; (b) P02E/P103E gradient; (c) P04A/P103E gradient; (d) P04E/P103E gradient; inset) film thickness ( $\mu$ m) vs \% P04E.
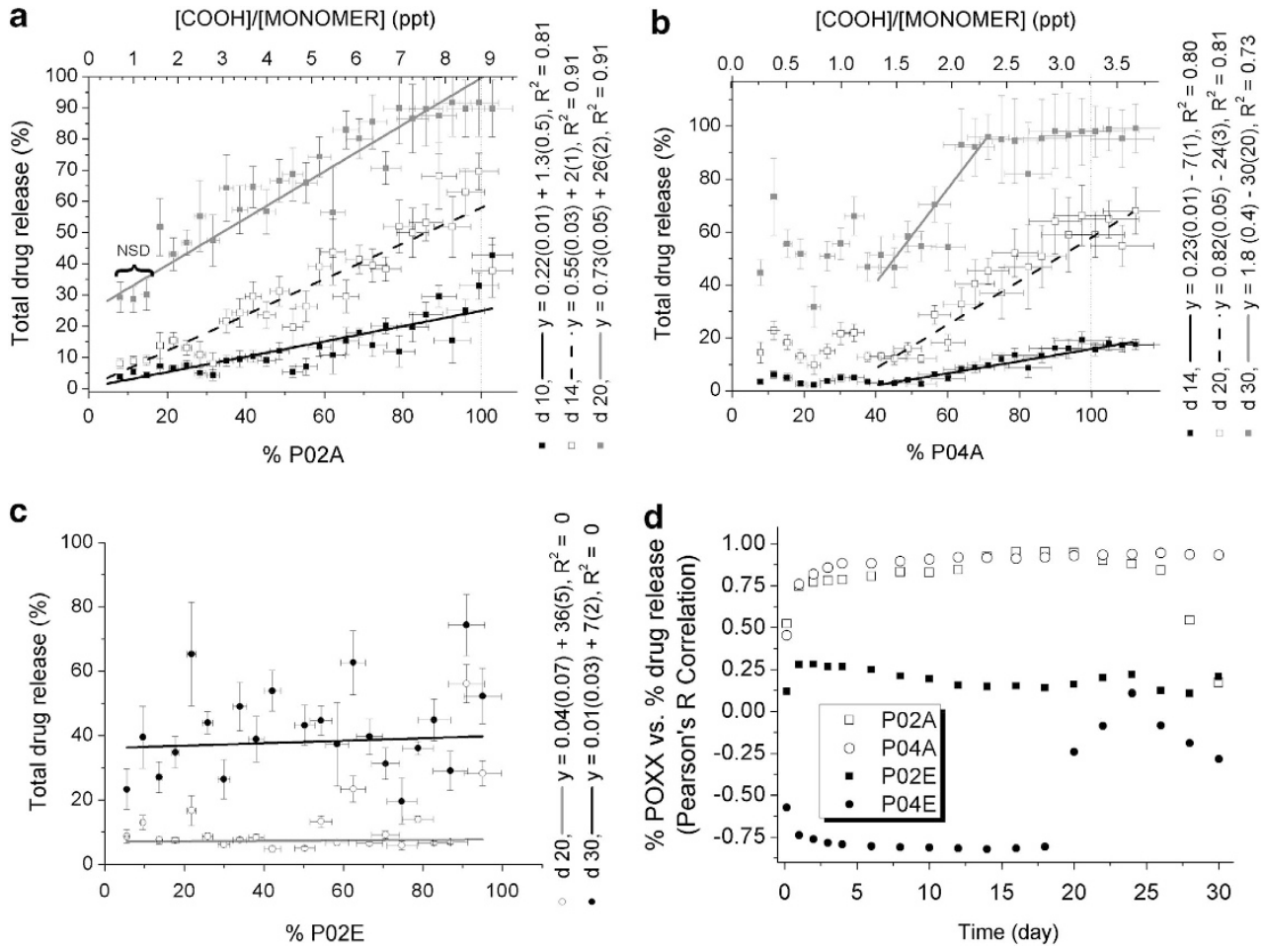

Figure 5 Total drug release as a function of acid terminal functional group at certain time points. (a) \%PO2A as a function of total drug release at 10 , 14, and 20 day. p.p.t., parts per thousand monomer lactide/glycolide. (b) \%PO4A as a function of total drug release at 14, 20 and 30 days. (c) \%P04E as a function of total drug release at 20, and 30 days. (d) Pearson's $r$ correlation of the \% POXX additive as a function of the \% total drug release. 
percent P02A had R-square values of 0.8, 0.9 and 0.9, respectively. The data suggest that at day 20, a formulation with P02A can be predicted to achieve drug release of from 40 to $90 \%$. However, not every concentration of polyesters was effective in modulating the release. The acid concentration was found to be effective in modulating drug release only above certain concentrations. At a threshold below $\sim 1$ p.p.t. of $[\mathrm{COOH}] /[\mathrm{MONOMER}]$, no trends were observed for the $\mathrm{P} 02 \mathrm{~A}$ or P04A polyesters.

\section{DISCUSSION}

To control rates of drug delivery in biodegradable polymer matrices, one must modify one or more of the four generally accepted release mechanisms: (1) water-saturated pore diffusion, (2) polymer matrix diffusion, (3) osmotic gradients or (4) polymer matrix erosion. ${ }^{15}$ The first two mechanisms are based on diffusion and rely on fastdissolving additives to increase pore networks. These additives are sometimes referred to as 'porogens'. These systems are best suited for hydrophilic therapeutics, but they often exhibit substantial burst release and generally negatively affect the material properties of the drug delivery system. ${ }^{18}$ For hydrophobic drug molecules, they tend to partition strongly to the polymer matrix, with diffusion playing a minor role in their release (nanoparticles being an exception). The hydrophobic drugs only release when the polymer fragments or amphiphilic additives diffuse from the matrix. ${ }^{4,19,20}$ Recent investigations of PLGA erosion support this view. PLGA mass loss was largely dependent on the solubility of the degraded fragments, not on diffusion-controlled reactions. ${ }^{21}$ PLGA fragments were reported to be soluble after a critical point of $15000 \mathrm{Da}$, but are typically soluble at $\sim 1000 \mathrm{Da}^{21-23}$ Therefore, to control the drug delivery of hydrophobic compounds, one must find mechanisms to control the rates of polyester erosion.

Blending of polyesters using carboxylic acid end-groups has previously been reported for microsphere formulations. ${ }^{9-12}$ Acidic groups in polymer matrices are known to catalyze the degradation of polyester polymers. The aggregate results from these microsphere formulations suggested that carboxylic acid end-groups can be used to tailor or modulate the delivery of hydrophobic drugs. Owing to the laborious nature of most drug release protocols, no studies have evaluated the statistical correlations between the amount of acid endgroups and controlled drug release, especially for thin films incorporating hydrophobic drugs for long-term release. With our recent development of high-throughput quantitation and film gradient-casting procedures, drug release profiling of various thin films is now feasible. ${ }^{6,13}$

We synthesized increasingly acidic gradient films having concentrations of $0-3.3$ and $0-8.9$ p.p.t. [COOH]/[MONOMER] carboxylic acid terminal end-groups with the usage of commercially available $\mathrm{P} 04 \mathrm{~A}$ and $\mathrm{P} 02 \mathrm{~A}$ blended into $\mathrm{P} 103 \mathrm{E}$, respectively. Complete chromatographic separation of the mixed polymers was not possible by size exclusion chromatography. Therefore, a deconvolution analysis was performed, as shown in Figure 1. By having two sets of gradient concentrations, a fine-tuning and extended range acidic analysis could be performed. However, this analysis also set up polymer MW gradients that were proportional to the acid terminal end-groups. Gradients of P04E and P02E were used as controls that had similar MW gradients but no acidic gradients, as demonstrated in Figure 2. To limit drug release by diffusion, a small $1 / 40 \mathrm{w} / \mathrm{w}$ ratio of fluorescent drug/polymer was implemented. The concentrations of FDAc compared with the polymer constituents are shown in Figure 3. Therefore, the controlled drug release was mostly dependent on PLGA erosion.
The results in Figures 4 and 5 display the effects of the acid vs ester terminal groups on the drug release of FDAc, which is a molecule that mimics the release of paclitaxel and allows high-throughput drug release. ${ }^{13}$ Using acid end-groups, we were able to tailor a wide range of drug release formulations. For P02A at day 14 and P04A at day 20, a $10-60 \%$ range of drug release could easily be predicted and was linearly dependent on the acid end-group concentration. Although these results are for small molecule hydrophobic drugs, previous investigations by Raiche and Puleo ${ }^{9}$ suggested that tunable ranges for protein release are feasible through acid gradients. The P04A gradients displayed a short range of tunable drug release because of the small acidic concentration range. However, P04A gradients allowed a better estimate of the threshold of acidic groups required to accelerate controlled drug release. Figures $4 \mathrm{c}$ and $5 \mathrm{~b}$ show that increased drug release was not observed below $40 \%$ P04A or below $\sim 1.2$ p.p.t. acidic end-groups. Similar results were obtained for $<15 \%$ P02A, as shown in Figure 5a. In this regard, molecular mass also had a significant effect on drug release. However, we can provide no predictive correlation when drug release was compared with varying MW PLGA with the same acid terminal group. The results must be viewed within the limitations of the two MWs tested, where the range was determined with 20 and $50 \mathrm{kDa}$. More ranges are required for statistical correlations.

If controlled drug release is directly dependent on the PLGA degradation, this acidic threshold concentration is significant for a number of polyester medical implant processes, such as injection molding, plasma surface modifications and irradiation for sterilization. If not strictly controlled, all of these manufacturing steps cause polyester degradation and acidic end-group formation. ${ }^{24-28}$ If the above processes increase the acidic residues to $>1$ p.p.t. in the PLGA thin films, our results predict an increase in erosion within 30 days.

Alternatively, the linear dependence of the increase in acid endgroups on the total drug release may allow drug delivery implants to dynamically change their delivery rates in vivo via specific event triggers. For example, several technologies used by the photolithography industry allow instantaneous formation of carboxylic acid groups through the conversion of ortho-nitrobenzyl esters. ${ }^{29}$ Pulses of light create more carboxylic acid end-groups and would allow a gradual increase in drug delivery as needed. Similar formation of acidic end-groups can be envisioned by other 'SMART' triggered events (for example, ultrasound, electrical signal, light, temperature ${ }^{30}$ ) for switchable hydrophobic/hydrophilic surfaces. ${ }^{31}$ This would allow modulation of drug release, and after the drug reservoir is exhausted, subsequent triggers would allow the forced erosion of medical implants, resulting in faster clearance. We will be focusing on this area in our future studies.

We speculate that the dynamic use of acidic terminal functional groups would be applicable to a large number of other implantable polymers and macro-therapeutics. For example, a close polyester relative of PLGA (that is, PLLA) has often been employed for use in stents because of its strong material properties as well as its biodegradability. However, PLLA implants often take years to bioresorb (along with other polyesters like poly-caprolactone, polysebacic acid and other poly-alkanoate acids). ${ }^{32,33}$ For longer implant times, the development costs are higher because of animal testing and the long-term risks for inflammation and thrombosis also increase. Degradation times in years exceed the timeframes of how long a stent is actually needed, which is approximately 6 months. ${ }^{34}$ By balancing the PLLA MW and the activation of acidic groups within the matrix, one could optimize the material properties for use as a fast resorbable implant. 
1 Li, Y., Rodrigues, J. \& Tomas, H. Injectable and biodegradable hydrogels: gelation biodegradation and biomedical applications. Chem. Soc. Rev. 41, 2193-2221 (2012).

2 Mundargi, R. C., Babu, V. R., Rangaswamy, V., Patel, P. \& Aminabhavi, T. M. Nano/ micro technologies for delivering macromolecular therapeutics using poly(D,L-lactideco-glycolide) and its derivatives. J. Control. Release 125, 193-209 (2008).

3 Rytting, E., Nguyen, J., Wang, X. \& Kissel, T. Biodegradable polymeric nanocarriers for pulmonary drug delivery. Expert Opin. Drug Deliv. 5, 629-639 (2008).

4 Steele, T. W., Huang, C. L., Widjaja, E., Boey, F. Y., Loo, J. S. \& Venkatraman, S. S. The effect of polyethylene glycol structure on paclitaxel drug release and mechanical properties of PLGA thin films. Acta Biomater. 7, 1973-1983 (2011).

5 Vlachou, M., Hani, N., Efentakis, M., Tarantili, P. A. \& Andreopoulos, A. G. Polymers for use in controlled release systems: the effect of surfactants on their swelling properties. J. Biomater. Appl. 15, 65-77 (2000)

6 Steele, T. W. J., Huang, C. L., Kumar, S., Irvine, S., Chiang Boey, F. Y., Loo, J. S. C. \& Venkatraman, S. S. Novel gradient casting method provides high-throughput assessment of blended polyester poly(lactic-co-glycolic acid) thin films for parameter optimization. Acta Biomater. 8, 2263-2270 (2012).

7 Ishihara, T., Kubota, T., Choi, T., Takahashi, M., Ayano, E., Kanazawa, H. \& Higaki, M. Polymeric nanoparticles encapsulating betamethasone phosphate with different release profiles and stealthiness. Int. J. Pharm. 375, 148-154 (2009).

8 Tang, Z. G. \& Hunt, J. A. The effect of PLGA doping of polycaprolactone films on the control of osteoblast adhesion and proliferation in vitro. Biomaterials 27, 4409-4418 (2006).

9 Raiche, A. T. \& Puleo, D. A. Modulated release of bioactive protein from multilayered blended PLGA coatings. Int. J. Pharm. 311, 40-49 (2006).

10 Duvvuri, S., Gaurav Janoria, K. \& Mitra, A. K. Effect of polymer blending on the release of ganciclovir from PLGA microspheres. Pharm. Res-Dordr. 23, 215-223 (2006).

11 Panyam, J., Williams, D., Dash, A., Leslie-Pelecky, D. \& Labhasetwar, V. Solid-state solubility influences encapsulation and release of hydrophobic drugs from PLGA/PLA nanoparticles. J. Pharm. Sci. 93, 1804-1814 (2004).

12 Su, Z., Sun, F., Shi, Y., Jiang, C., Meng, Q., Teng, L. \& Li, Y. Effects of formulation parameters on encapsulation efficiency and release behavior of risperidone poly $(D, L$ lactide-co-glycolide) microsphere. Chem. Pharm. Bull. (Tokyo) 57, 1251-1256 (2009).

13 Steele, T. W., Huang, C. L., Kumar, S., Widjaja, E., Chiang Boey, F. Y., Loo, J. S. \& Venkatraman, S. S. High-throughput screening of PLGA thin films utilizing hydrophobic fluorescent dyes for hydrophobic drug compounds. J. Pharm. Sci. 100, 4317-4329 (2011).

14 Huang, C. L., Kumar, S., Tan Jun Zhi, J., Boey, F. Y., Venkatraman, S. S., Steele, T. W. \& Loo, J. S. Modulating drug release from poly(lactic-co-glycolic acid) films through terminal end-groups and molecular weight. Polym. Degrad. Stabil. 98, 619-626 (2013).

15 Fredenberg, S., Wahlgren, M., Reslow, M. \& Axelsson, A. The mechanisms of drug release in poly(lactic-co-glycolic acid)-based drug delivery systems-a review. Int. J. Pharm. 415, 34-52 (2011).

16 Jonnalagadda, S. \& Robinson, D. H. Effect of thickness and PEG addition on the hydrolytic degradation of PLLA. J. Biomaterials Sci. Polym. Edn. 15, 1317-1326 (2004)

17 Fortier-McGill, B., Toader, V. \& Reven, L. H-1 solid state NMR study of poly(methacrylic acid) hydrogen-bonded complexes. Macromolecules 45, 6015-6026 (2012).

18 Glaessl, B., Siepmann, F., Tucker, I., Rades, T. \& Siepmann, J. Deeper insight into the drug release mechanisms in Eudragit RL-based delivery systems. Int. J. Pharm. 389 139-146 (2010).
19 Kang, J. C. \& Schwendeman, S. P. Determination of diffusion coefficient of a small hydrophobic probe in poly(lactide-co-glycolide) microparticles by laser scanning confocal microscopy. Macromolecules 36, 1324-1330 (2003).

20 Mittal, G., Sahana, D. K., Bhardwaj, V. \& Kumar, M. N. V. R. Estradiol loaded PLGA nanoparticles for oral administration: effect of polymer molecular weight and copolymer composition on release behavior in vitro and in vivo. J. Control. Release 119, 77-85 (2007).

21 Korber, M. PLGA erosion: solubility- or diffusion-controlled? Pharm. Res-Dordr. 27, 2414-2420 (2010).

22 Park, T. G. Degradation of poly(D,L-lactic acid) microspheres - effect of molecularweight. J. Control. Release 30, 161-173 (1994).

23 Husmann, M., Schenderlein, S., Luck, M., Lindner, H. \& Kleinebudde, P. Polymer erosion in PLGA microparticles produced by phase separation method. Int. J. Pharm. 242, 277-280 (2002).

24 Chia, N. K., Venkatraman, S. S., Boey, F. Y. C., Cadart, S. \& Loo, J. S. C. Controlled degradation of multilayered poly(lactide-co-glycolide) films using electron beam irradiation. J. Biomed. Mater. Res. Part A 84A, 980-987 (2008).

25 Ghosh, S., Viana, J. C., Reis, R. L. \& Mano, J. F. Effect of processing conditions on morphology and mechanical properties of injection-molded poly(L-lactic acid). Polym Eng. Sci. 47, 1141-1147 (2007).

26 Koo, G. H. \& Jang, J. Surface modification of poly(lactic acid) by UV/Ozone irradiation. Fiber. Polym. 9, 674-678 (2008).

27 Martinez-Sancho, C., Herrero-Vanrell, R. \& Negro, S. Study of gamma-irradiation effects on aciclovir poly(D,L-lactic-co-glycolic) acid microspheres for intravitreal administration. J. Control. Release 99, 41-52 (2004).

28 Han, Y. R., Jin, X. Y., Yang, J., Fan, Z. Y., Lu, Z. Q., Zhang, Y. \& Li, S. M. Totally bioresorbable composites prepared from poly(L-lactide)-co-(trimethylene carbonate) copolymers and poly(L-lactide)-co-(glycolide) fibers as cardiovascular stent material. Polym. Eng. Sci. 52, 741-750 (2012).

29 Klinger, D. \& Landfester, K. Polymeric photoresist nanoparticles: light-induced degradation of hydrophobic polymers in aqueous dispersion. Macromol. Rapid Comm. 32, 1979-1985 (2011).

30 Moon, H. K., Son, M., Park, J. E., Yoon, S. M., Lee, S. H. \& Choi, H. C. Significant increase in the water dispersibility of zinc phthalocyanine nanowires and applications in cancer phototherapy. NPG Asia Mater. 4, e12 (2012).

31 Zhang, L. B., Zhang, Z. H. \& Wang, P. Smart surfaces with switchable super oleophilicity and superoleophobicity in aqueous media: toward controllable oil/water separation. NPG Asia Mater. 4, E8 (2012).

32 Shazly, T., Kolachalama, V. B., Ferdous, J., Oberhauser, J. P., Hossainy, S. \& Edelman, E. R. Assessment of material by-product fate from bioresorbable vascular scaffolds. Ann. Biomed. Eng. 40, 955-965 (2012).

33 Gogotov, I. N., Gerasin, V. A., Kniazev, ,. V., Antipov, E. M. \& Barazov, S. [Composite biodegradable materials based on polyhydroxyalkanoate]. Prikl. Biokhim. Mikrobiol. 46, 659-665 (2010).

34 Ormiston, J. A. \& Serruys, P. W. Bioabsorbable coronary stents. Circ. Cardiovasc. Interv. 2, 255-260 (2009).

(c) (i) $\odot$ This work is licensed under a Creative Commons Attribution-NonCommercial-NoDerivs 3.0 Unported License. To view a copy of this license, visit http://creativecommons. org/licenses/by-nc-nd/3.0/ 